\title{
Variabilidad en la micro-dosimetría beta del sedimento como principal causa de dispersión en la datación OSL de los depósitos fluviales costeros fini-pleistocenos conservados en la Cuenca del Río Mero (A Coruña, Galicia, España)
}

Sediment Beta-microdose variability as main cause of dispersion in OSL-quartz dating of Upper-Pleistocene coastal fluvial-deposits preserved at Mero-River Basin (A Coruña, Galicia, Spain)

\author{
CARLOS ARCE-CHAMORRO*11, JORGE SANJURJO-SÁNCHEZ ${ }^{1}$ \\ ${ }^{1}$ Unidade de Xeocronoloxía- Instituto Universitario de Xeoloxía, Universidade da Coruña. ESCI, \\ Campus de Elviña. 15071, A Coruña, España. \\ *Autor de contacto: espetenc@hotmail.com
}

https://doi.org/10.17979/cadlaxe.2020.42.0.7308

Recibido: 23/10/2020, Aceptado: 19/11/2020

\begin{abstract}
In this work, we have estimated the equivalent dose (De) from the OSL signal from quartz, for fluvial deposits of the Upper Pleistocene in the Mero River basin (A Coruña, Galicia, Spain) and preserved in the Ria of Coruña coastal margin (NW Iberian Peninsule). Such deposits show De distributions more scattered than expected, providing unexpected
\end{abstract}


high over-dispersion percentages of the mean (OD> 20\%). These values are usually correlated to incomplete-bleaching of the OSL signal due to the transport of quartz grains under turbulent conditions as a consequence of high suspended sediment loads. However, both distribution plots and normality-test show normal, symmetric and central distributions. No evidence of two or more populations in aliquots due to two groups of grains, namely y (i) one group of grains with well-bleached signals before the last burial event and (ii) another group of grains which an inherited signal from a previous burial episode. Moreover, DoseRecovery experiments on quartz grains show that the high dispersion is due to external and not internal factors. Thus, we have analyzed the activity concentration of radioisotopes in samples and the concentration of potassium in several grain-sizes, to assess if the origin of over-dispersion is microdosimetry caused by ${ }^{40} \mathrm{~K}$ from potassium, given the low dose rates (DR) measured in samples. Results show that this is the most probable cause of dispersion, and no evidence of partial bleaching is found.

Key words: Fluvial sediments; NW Iberian Peninsule; quartz OSL; Over-dispersion; Partial Bleaching; Beta micro-dosimetry; Potassium distribution.

\section{Resumen}

En este trabajo se han estudiado las dosis equivalentes (De) obtenidas de la señal OSL del cuarzo, para depósitos fluviales del Pleistoceno Superior de la cuenca del río Mero (A Coruña, Galicia, España) y conservados en el margen costero de la Ría de A Coruña (NO Península Ibérica). Estas De muestran distribuciones con una dispersión mayor a la esperada, obteniéndose porcentajes de sobre-dispersión elevados (OD $>20 \%$ ). Estos valores suelen asociarse al blanqueamiento incompleto de la señal OSL, debido al transporte de los granos de cuarzo en un medio turbio, debido una caga finos en supresión. No obstante, los gráficos y test de bondad de ajuste describen distribuciones normales, simétricas y centradas en la media. Tampoco indican evidencias de dos o más poblaciones de alícuotas conformadas por dos clases de granos de cuarzo agrupadas: (i) un grupo donde la señal de los granos ha sido bien blanqueada antes del último episodio de deposición y enterramiento y (ii) otro grupo que muestran señal heredada (residual). Sin embargo, los experimentos de Dose Recovery realizados indican que la dispersión se debe a factores extrínsecos y no intrínsecos al tipo de cuarzo estudiado. Por ello, se han analizado las concentraciones de radioisótopos en las muestras y la concentración de potasio en diferentes granulometrías, para estimar si el origen de la sobre-dispersión es la micro-dosimetria causada por el ${ }^{40} \mathrm{~K}$ del potasio, dada las bajas tasas de radiación y dosis anual medidas en las muestras. Los resultados obtenidos muestran que esta es la causa más probable de la dispersión, no encontrándose evidencias de blanqueamiento parcial.

Palabras clave: Depósitos fluviales; NO Península Ibérica: Señal OSL; Sobre-dispersión; Blanqueamiento incompleto; Micro-dosimetría beta; Distribución de Potasio. 


\section{INTRODUCCIÓN}

La luminiscencia estimulada ópticamente (OSL) permite calcular la edad de formación de sedimentos siliciclásticos a partir de las estimaciones de la dosis equivalente (De) y de la tasa de radiación natural (DR) (AITKEN, 1998). La De se estima en Grays (Gy) a partir de la señal OSL de granos de cuarzo y valores de menos de 1 Gy hasta unos 200 Gy permiten obtener edades en el rango de pocos siglos hasta 100-200ka, respectivamente. No obstante, cuando las tasas de radiación (DR) son elevadas puede ocurrir que la señal de saturación se alcance en pocos miles de años (BURGIDGE et al., 2006), limitando la edad máxima que alcanza la OSL (SINGARAYER AND BAILEY 2003; WINTLE AND MURRAY, 2006) a unas pocas decenas de miles de años. La saturación de la señal OSL por DR elevadas puede suponer una limitación para la datación en el en el NW de la Península Ibérica (SANJURJOSÁNCHEZ AND VIDAL-ROMANÍ, 2013) debido a la abundancia de elementos radiactivos como el ${ }^{40} \mathrm{~K}$, U y Th en rocas granitoides (BOYLE, 1982; GASCOYNE, 1992; CSN, 2000). Incluso en Galicia, a dosis más bajas y para sedimentos más antiguos, se han obtenido niveles de próximos a saturación para muestras fluviales y eólicas en torno a 50ka (ARCE-CHAMORRO, 2017).

La técnica OSL permite datar el último momento de enterramiento de granos de cuarzo. Una asunción básica es que, para estimar la De, esta señal OSL del cuarzo haya sido blanqueada completamente por la luz del sol durante los procesos previos de transporte, lo que habitualmente se produce en unos pocos segundos de exposición
(DULLER, 2004). Una vez depositados los granos de cuarzo y protegidos de la luz, la radiación natural del medio (DR) genera la señal OSL que proporcionará la edad del sedimento. No obstante, en depósitos fluviales con condiciones de transporte en medio acuoso y elevada turbidez, el grado de blanqueamiento de la señal puede ser incompleto (RITTENOUR, 2008; DULLER, 2008). Cuando esto ocurre, en el sedimento existe una mezcla de granos de cuarzo con la señal generada durante el tiempo de enterramiento y una señal heredada que se sumará a la anterior, aspecto que implica un riesgo de sobre-estimación de la De y de la edad (OLLEY ET AL., 1999).

Para estimar la De se pueden utilizar dos métodos: medir alícuotas individuales compuestas de varios granos de cuarzo (alícuotas multigrano o MG) o medir granos de cuarzo individuales o granos simples (denominado single-grain o SG). Si el blanqueado durante el transporte de los granos ha sido completo, la distribución de las estimaciones individuales de la De -a partir de cada MG o SG para una muestra dadaes de tipo normal, simétrica y centrada en estimadores como la media aritmética, la mediana o la media ponderada por el error a partir del Modelo de Edad Central (CAM) (GALBRAITH ET AL., 1999). No obstante, cuando en estas distribuciones se observa asimetría, amplia dispersión y la presencia de dos o más poblaciones, la estimación de la De promedio mediante el modelo CAM puede resultar inadecuada para estimar la edad, ya que tenderá a sobreestimarla. Para evaluar la dispersión se pueden utilizar varios indicadores. El principal es la denominada sobre-dispersión de la edad central, que es un estimador de la varianza observada respecto a la esperada para 
una determinada distribución. Este valor no debería superar el 20-30\% (OLLEY ET AL., 1999). Cuando se supera, suele recomendarse el uso de otros modelos, dentro de los cuales el más frecuente es el Modelo de Edad Mínima (MAM; GALBRAITH ET AL., 1999) o el modelo IEU (THOMSEN ET AL., 2003), éste último solo aplicable a SG. En el modelo MAM, se considera que la población de MG o SG con menor De corresponde a granos bien blanqueados, que a su vez pertenecen a una distribución normal truncada en los valores más bajos a partir de la cual se estima un valor promedio ponderado con su error. En este caso, la selección del modelo de edad para estos sedimentos fluviales pasa por un análisis estadístico de la distribución a partir de histogramas, diagramas de cajas, radial-plots o los test de normalidad -aunque se están desarrollando otros medios como análisis de la señal OSL o técnicas combinadas de señal OSL de y pIR-IRSL de feldespato de una misma muestra, para ampliar el conocimiento en este campo (BUCKLAND ET AL., 2019).

Existen otros factores extrínsecos que pueden causar una elevada sobredispersión. Uno de ellos está relacionado con una distribución heterogénea de radio-isótopos en la matriz sedimentaria estudiada, afectando principalmente a la radiación beta (NATHAN ET AL., 2003) debido a su menor poder de penetración $(0.2 \mathrm{~cm})$-respecto a la radiación gamma $(30 \mathrm{~cm})$. A escala centimétrica, la tasa de radiación beta será significativamente menor en aquellas áreas con un contenido bajo radioisótopos, respecto a aquellas áreas con mayor proporción de radioisótopos. Así, los granos de cuarzo situados en una y otra zona experimentarán tasas de radiación beta diferentes. Esto es especialmente frecuente cuando las tasas de radiación beta son bajas y en el caso del ${ }^{40} \mathrm{~K}$ presente en feldespato potásico. Los granos de cuarzo cercanos a granos feldespato potásico se verán afectados por la radiación beta del ${ }^{40} \mathrm{~K}$ de estos últimos (hot-spots). Esta heterogeneidad se denomina variabilidad en la micro-dosimetría beta (DR-BETA) y genera dispersión cuando se mide la OSL en SG o MG.

Por último, cabe considerar la contribución de los factores intrínsecos, pudiendo afectar a la precisión de las De estimada. Uno de ellos es sensibilidad a la luminiscencia de los propios granos de cuarzo analizados, en relación a la intensidad de su señal OSL (THOMSEN ET AL., 2003; 2005) y su respuesta a la irradiación artificial en laboratorio, tratamientos térmicos y a la estimulación lumínica en el laboratorio al utilizar el protocolo SAR (Single Aliquot Regenerative-dose) (MURRAY AND WINTLE, 2000). Cuando la sensibilidad a la estimulación óptica es baja, las señales son poco intensas y tienen una baja relación señal/fondo, pudiendo derivar en Des imprecisas. A este efecto, el modo aceptado de evaluar la idoneidad del protocolo SAR empleado para la estimación de la De es a partir del denominado test Dose-Recovery (WALLINGA ET AL., 2000). Para este test se blanquea completamente la señal OSL de un determinado número de alícuotas MG o SG preparados. El blanqueado se puede realizar exponiendo la alícuota a la luz del sol o a LEDs en el lector de luminiscencia, eliminando así cualquier señal heredada por el fenómeno de blanqueamiento incompleto. Posteriormente, a cada alícuota (MG o $\mathrm{SG})$ se les induce una dosis de radiación beta conocida, próxima a la De estimada. 
Como esta dosis artificial es homogénea, se anula cualquier tipo de incertidumbre derivada de variaciones (extrínsecas) como la micro-dosimetría, bio o crioturbación o la señal heredada cuando se produce blanqueamiento incompleto. Por último, se procede a la estimación de esta dosis artificial mediante el protocolo SAR, en base a los criterios de aceptación establecidos. Este test ofrece información muy valiosa debido a que la incertidumbre asociada a estas estimaciones sólo es atribuible a factores intrínsecos, resultando un método eficaz para cuantificar su contribución al porcentaje de sobre-dispersión. Así, el modelo de edad a utilizar para la estimación de la dosis equivalente al tiempo de enterramiento y formación de estos depósitos fluviales, pasa por el análisis y conocimiento de las fuentes de incertidumbre asociada a unos resultados muy variables y dispersos, como los observados en el presente trabajo.

\section{OBJETIVO}

El objetivo principal es analizar qué factores (intrínsecos y/o extrínsecos) están contribuyendo al incremento de la incertidumbre en aquellas muestras afectadas por valores de sobre-dispersión por encima de lo esperado, considerando que son sedimentos fluviales antiguos desarrollados sobre los esquistos alterados, a efectos de tasa de radiación natural, presencia potencial de granos de cuarzo con blanqueamiento incompleto y presencia de potasio en el sedimento. Para ello se realizará un análisis estadístico de la distribución de datos, un análisis de la sobre-dispersión respecto a la media ponderada del CAM y del test Dose-Recovery, así como un análisis de la distribución de potasio (como fuente de ${ }^{40} \mathrm{~K}$ y radiación beta del sedimento) en las distintas fracciones granulométricas y su correlación con la DR, DRBeta y sobre-dispersión estimada $(\% \mathrm{OD})$. 


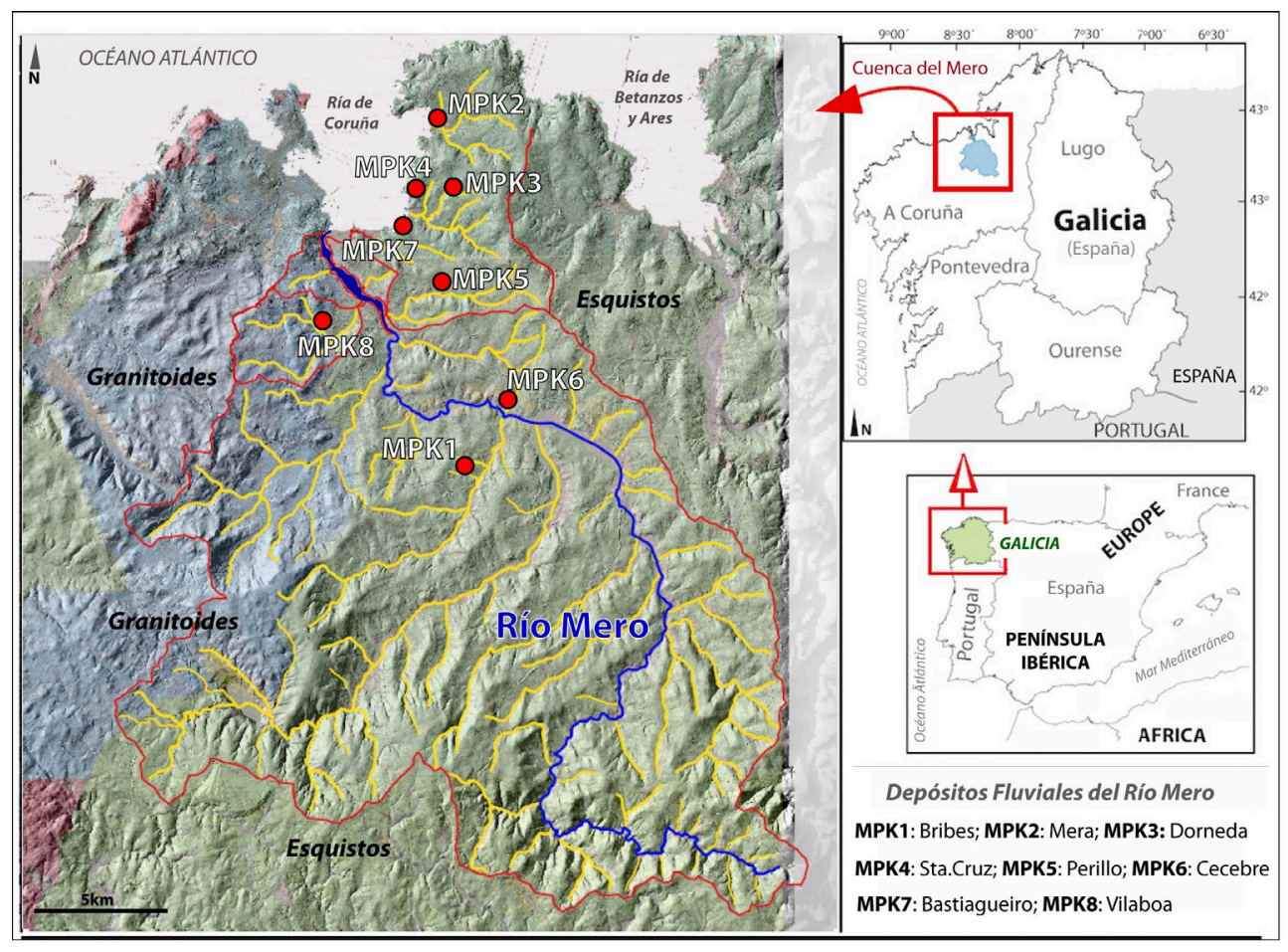

Figura 1. Mapa de situación de la Cuenca del Río Mero: Todos los sedimentos fluviales se desarrollan a partir de litología esquistosa (áreas en verde) a excepción de la muestra MPK8 (Vilaboa), desarrollada a partir de litología granitoide (áreas en azul), sin que ello implique variaciones en la DR estimada. (MDT05-IGME y Mapa geológico IGME-MAGNA50, hojas 21, 22, 45, 46, 70 y 70: http://centrodedescargas.cnig.es/; Hidrología: Augas de Galicia (http://mapas.xunta.gal/xeoportal)).

\section{3. ÁREA DE ESTUDIO}

El presente trabajo es el resultado de la datación mediante OSL de un sistema de depósitos fluviales seleccionados en la cuenca del Río Mero (ARCE-CHAMORRO, 2017), ricos en arenas y limos, próximos a la costa o dentro del propio litoral de la Ría de Coruña (A Coruña, Galicia, España) (Fig.1). Siete de los ocho depósitos se han desarrollado a partir de litología esquistosa de alto grado de alteración
(ENGELS, 1974; ARENAS ET AL., 2000), correspondientes a las muestras de Dorneda (43⒉ $\left.26^{\prime \prime} \mathrm{N} ; 8^{\circ} 19^{\prime} 52^{\prime \prime} \mathrm{O}\right)$, Santa Cruz (43⒉ $\left.51^{\prime \prime} \mathrm{N} ; 8^{\circ} 20^{\prime} 18^{\prime \prime} \mathrm{O}\right)$ (Fig.2),

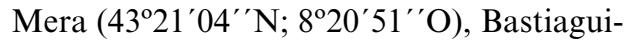
ero $\left(43^{\circ} 20^{\prime} 27^{\prime \prime} \mathrm{N}\right.$; $\left.8^{\circ} 20^{\prime} 57^{\prime \prime} \mathrm{O}\right)$, Mesón de Auga-Perillo $\quad\left(43^{\circ} 19^{\prime} 27^{\prime \prime} \mathrm{N} ; \quad 8^{\circ} 20^{\prime} 18^{\prime \prime} \mathrm{O}\right)$ (Fig.2), Bibres $\left(43^{\circ} 16^{\prime} 33^{\prime \prime} \mathrm{N}\right.$; $\left.8^{\circ} 18^{\prime} 46^{\prime \prime} \mathrm{O}\right)$ y Cecebre $\left(43^{\circ} 17^{\prime} 29^{\prime \prime} \mathrm{N}\right.$; $\left.8^{\circ} 18^{\prime} 37^{\prime \prime} \mathrm{O}\right)$ - y uno a partir de granitoides (BASTIDA ET AL., 1984) -muestra Vilaboa $\left(43^{\circ} 18^{\prime} 45^{\prime \prime} \mathrm{N}\right.$; $8^{\circ} 23^{\prime} 01^{\prime \prime} \mathrm{O}$ ). 

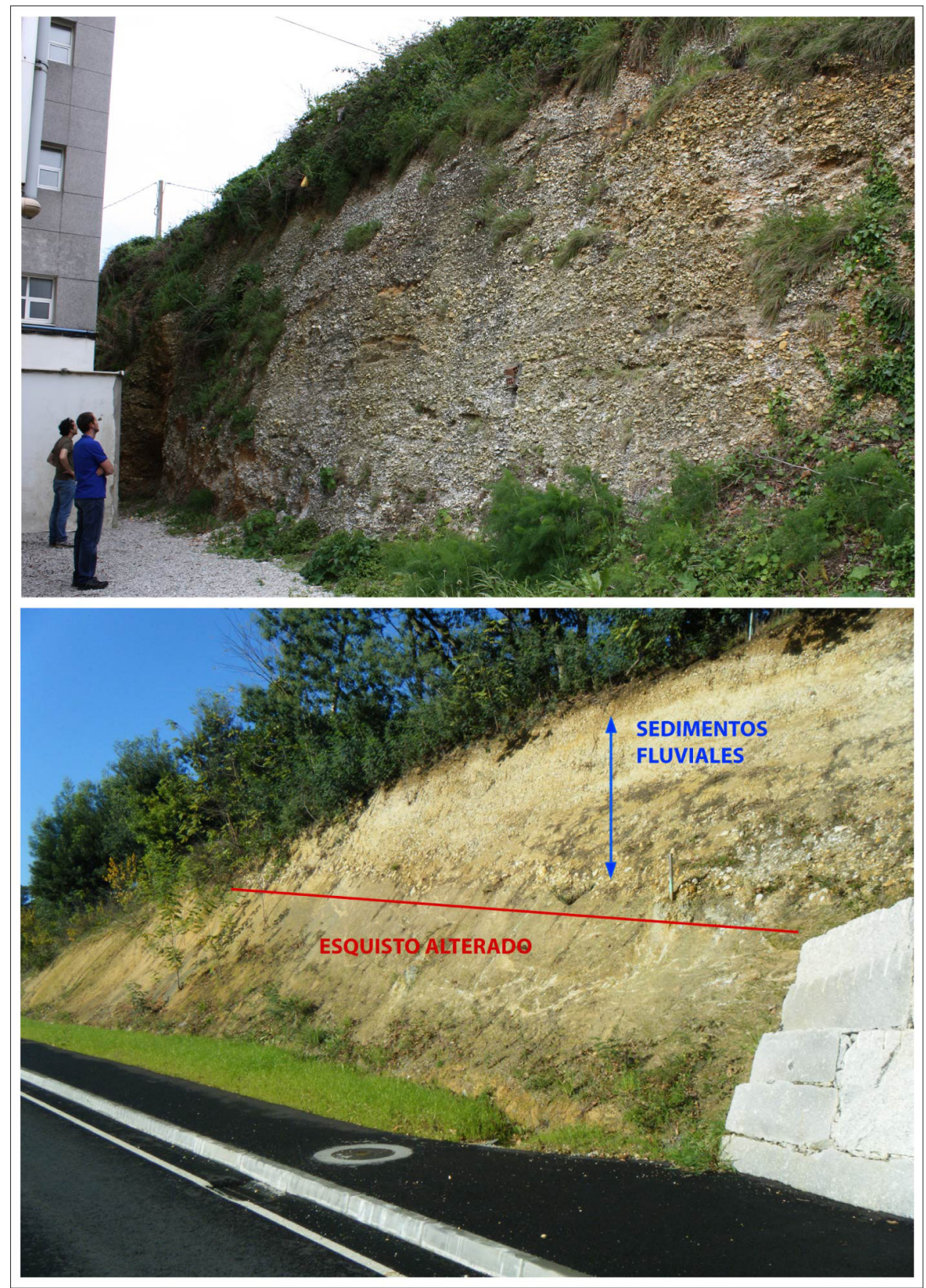

Figura 2. (Arriba): Fotografía del depósito fluvial de Santa Cruz (Oleiros, A Coruña) con la siempre grata presencia del Doctor en Geología Viveen Willem, durante una jornada de campo. (Abajo): Fotografía del depósito fluvial de Perillo (Oleiros, A Coruña), desarrollado sobre esquistos alterados. 


\section{MATERIAL Y MÉTODOS}

Las muestras se han extraído a partir de testigos de acero $(1 \mathrm{~cm}$ de grosor, $30 \mathrm{~cm}$ de largo y $10 \mathrm{~cm}$ de diámetro) en áreas homogéneas. En condiciones de seguridad lumínica (LEDs rojos) se tamiza en seco la fracción más adecuada para datación OSL (180-250 $\mu \mathrm{m})$ (AITKEN, 1998; TRUELSEN AND WALLINGA, 2003) de la que se extraerán y purificarán los granos de cuarzo El primer paso es un lavado mediante ataque ácido $\left(10 \% \mathrm{HCl} ; 3 \mathrm{~h} ; 50^{\circ} \mathrm{C}\right)$ y ataque oxidativo $\left(10 \% \mathrm{H}_{2} \mathrm{O}_{2}\right)$. Después se extraen los granos de cuarzo mediante centrifugado en liquido ultra-denso (poli-tungsteno de sodio; $15 \mathrm{ml} / 5 \mathrm{~g}$ ): (i) centrifugado a densidad $2.62 \mathrm{~g} / \mathrm{cc}$, donde precipitan cuarzo y minerales pesado que se centrifugarán (ii) a densidad $2.70 \mathrm{~g} / \mathrm{cc}$, recogiendo los granos de cuarzo del sobrenadante. Cualquier impureza presente en estos granso de cuarzo (como feldespatos) se lava con fluorhídrico $(20 \%)$, tratamiento (HF-etching) que elimina la porción superficial de los granos de cuarzo afectada por la componente alfa $\left(\mathrm{D}_{\text {R-ALPHA }}\right.$ ) (AITKEN, 1998) y un $10 \%$ de la $\mathrm{D}_{\text {R-BETA }}$ (BRENAN, 2003).

La medida de la señal OSL se realiza con un lector OSL-IRSL Riso TL/OSL DA-15 (BÆTTER-JENSEN ET AL., 2010) con fuente de estímulo (LEDs azul y filtro de emisión de longitud de onda de $430 \mathrm{~nm}$ a $80 \mathrm{~mW} / \mathrm{cm}^{2}$ y LED infrarrojo de $870 \mathrm{~nm}$ a $135 \mathrm{~mW} / \mathrm{cm}^{2}$ ), filtros de detección (OSL Hoya U-340/7.5mm e IRSL Schott BG-39/ Cornig 7-59), tubo fotomultiplicador (PMT bialkali EMI 9235QB), fuente beta $\left({ }^{90} \mathrm{Sr} /{ }^{90} \mathrm{Y}\right)$ calibrada y placa térmica. Para ello se emplean alícuotas multigrano de 100 granos (aprox.) (DULLER, 2008; HEER ET AL., 2012) en discos de acero $(\varnothing=9.7 \mathrm{~mm})$ y una regleta de $2 \mathrm{~mm}$, analizadas mediante el protocolo SAR (Single Aliquot Regenerative-dose) (MURRAY AND WINTLE, 2000). Este protocolo incluye el test-dose para la normalización de la señal OSL natural y regeneradas (RHODES, 2000; WINTLE, 2008). Para seleccionar la temperatura idónea para el SAR se realiza un test de pre-heat (ROBERTS ET AL., 1994; MURRAY AND WINTLE, 1999) en un rango entre 180 to $280^{\circ} \mathrm{C}$. También se evalúa con el SAR la variabilidad de la señal OSL en función del tamaño de alícuota (dose-size aliquot test; RODNIGHT ET AL., 2006) a partir de alícuotas con diferentes tamaños $\left(\mathrm{N}^{\mathrm{o}}\right.$ granos $\left.=10,30,100,>300,1000\right)$.

El comportamiento de la componente rápida de la señal OSL (BAILEY ET AL., 1997; JAIN ET AL., 2003) se ha valorado en un test de blanqueamiento de la señal (Bleaching-test; PRZEGIETKA AND CHRUSCINSKA, 2013) -a distintos intervalos de blanqueamiento de la señal $(0.0 \mathrm{~s}$, $0.1 \mathrm{~s}, 0.2 \mathrm{~s}, 1 \mathrm{~s}, 2 \mathrm{~s}, 10 \mathrm{~s}, 100 \mathrm{~s}$ and 1000s). La señal OSL se ha integrado mediante el método LBG (Late Background) (BANERJEE ET AL., 2000) y mediante el método EBG (Early Background) (BALLARINI ET AL., 2007; CUNNINHAM AND WALLINGA, 2010), dentro de los criterios de señal-fondo aceptables (MURRAY AND WINTLE, 2000). Aquellas alícuotas que no superan los criterios de aceptación establecidos en el protocolo o aquellas cuya estimación de la De supere los 200Gy -valor establecido por convenio como límite del umbral de saturación de la señal- han sido rechazadas. El blanqueamiento de la señal OSL para el test Dose-Recovery (WALLINGA ET AL., 2000) se ha realizado a partir de LEDs azul e inducción de dosis beta de $84 \mathrm{~Gy}$, obteniendo resultados satisfactorios dentro de 
los criterios de aceptación. La estimación de la De y del porcentaje de sobre-dispersión (OD) se ha realizado mediante el modelo CAM (GALBRAITH ET AL., 1999). Los test de bondad de ajuste empleados son el de Kolmogorov-Smirnov (Lilliers.) y el de Saphiro-Wilks (si $\mathrm{n}<50)$.

La estimación de las componentes DRGAMMA y DR-BETA se ha realizado mediante espectrometría gamma de alta resolución (HRGe) a partir de $300 \mathrm{~g}$ gramos de muestra bruta procedente del hueco del testigo, deshidratada $\left(105^{\circ} \mathrm{C} ; 48 \mathrm{~h}\right)$, molida y tamizada por debajo de $63 \mu \mathrm{m}$, posteriormente almacenada durante un mes en una campana de desecación a temperatura ambiente para re-equilibrio del radón. La actividad de los radioisótopos de ${ }^{40} \mathrm{~K}$ y de las cadenas de ${ }^{238} \mathrm{U}$ y ${ }^{232} \mathrm{Th}$ (IVANOVICH AND HARMON, 1982) se ha realizado mediante los grupos de equilibrio de OCZKOWSKI ET AL. (2000). En base a los resultados se considera equilibrio en base a los grupos ${ }^{238} \mathrm{U} \rightarrow{ }^{226} \mathrm{Ra} \rightarrow{ }^{210} \mathrm{~Pb} \quad$ y $\quad{ }^{232} \mathrm{Th} \rightarrow{ }^{238} \mathrm{Ra} \rightarrow{ }^{228} \mathrm{Th}$ (OLLEY ET AL., 1999) para cada serie. La componente cósmica se ha calculado en base a la posición geográfica y la profundidad del muestreo con respecto a la superficie (PRESCOTT AND HUTTON, 1994).

El análisis granulométrico se ha realizado mediante granulometría láser $(0.1-250 \mu \mathrm{m})$ y tamizado húmedo $(250-2000 \mu \mathrm{m})$, aunando las fracciones gruesa $(63-<2000 \mu \mathrm{m})$ y fina $(<63 \mu \mathrm{m})$. También se ha realizado un análisis de proporción relativa de elementos mediante FRX para cada una de las fracciones correspondientes a arena gruesa $(1000-500 \mu \mathrm{m})$, media $(500-125 \mu \mathrm{m})$, fina $(125-63 \mu \mathrm{m})$, limo $(63-2 \mu \mathrm{m})$ y arcilla $(<2 \mu \mathrm{m})$ (WENTWORTH, 1922), tamizadas en seco, haciendo énfasis en el Potasio como aproximación a la proporción del radioisótopo ${ }^{40} \mathrm{~K}$.

\section{RESULTADOS Y DISCUSIÓN}

\subsection{Tasa de radiación natural (DR) y Se- ñal OSL.}

La tasa de radiación natural (DRTOTAL) estimada se desglosa en la Tabla 1 , presentando valores entre 0.68 y $1.83 \mathrm{~Gy} / \mathrm{ka}$. La muestra de Vilaboa-MPK8, desarrollada en una zona de granitoides, presenta una tasa de Gy/ka 1.64 $\pm 0.16 \mathrm{~Gy} / \mathrm{ka}$ por debajo de la muestra desarrollada en zona de esquistos de Santa-Cruz-MPK2 (1.83 \pm 0.14 Gy/ka), como valor superior. Estas tasas se sitúan en el rango inferior de las estimaciones de referencia a nivel regional, entre $1.4 \mathrm{y}$ hasta 7 Gy/ka (CUNHA ET AL., 2008; MARTINS ET AL., 2010; SANJURJO-SÁNCHEZ Y VIDAL-ROMANÍ, 2011; RAMOS ET AL., 2012; MEDIALDEA, 2013; VIVEEN ET AL., 2013; TRINIDADE ET AL., 2016; RIBEIRO ET AL., 2019) si bien, la mayoría de estas estimaciones de referencia se han realizado sobre materiales procedentes $\mathrm{O}$ pertenecientes a litologías graníticas. En el caso que nos ocupa, y aunque sólo se dispone de una única muestra desarrollada en la zona granítica, no se observan diferencias respecto a los esquistos. Las estimaciones de la componente DR-GAMMA varía entre 0.19 y $0.79 \mathrm{~Gy} / \mathrm{ka}$. Las de la componente DR-BETA entre 0.30 y $0.84 \mathrm{~Gy} / \mathrm{ka}$. Ambas contribuyen de forma similar, sin grandes diferencias entre dosis y litología. En cuanto a la actividad radioisotópica, se observa una mayor contribución del ${ }^{40} \mathrm{~K}$ (78 y 226 $\mathrm{Bq} / \mathrm{ka}$ ), si se compara con la actividad del ${ }^{238} \mathrm{U}$ (entre 16 y $26 \mathrm{~Bq} / \mathrm{ka}$ ) o el ${ }^{232} \mathrm{Th}$ (entre 10 y $36 \mathrm{~Bq} / \mathrm{ka}$ ) -la actividad del ${ }^{235} \mathrm{U}$ resulta insignificante (ARCE-CHAMORRO, 2017). Las tasas estimadas se corresponden, por tanto, a dosis bajas pero prolongadas en el tiempo. 
Para muestras antiguas, con dosis (DR) prolongadas en el tiempo, cabe esperar señales OSL próximas al umbral de saturación si bien, también cabe esperar señales OSL intensas al generarse, hipotéticamente, una mayor redistribución de cargas -acompañadas, además de una incertidumbre mayor, asociada a la estadística de cuentas, la sensibilidad y la respuesta de los materiales (ARNOLD AND ROBERTS, 2009). No obstante, la señal natural OSL $\left(\mathrm{L}_{\mathrm{N}}\right)$ en aquellas muestras procedentes de esquistos alterados presenta una intensidad muy baja $(<40.000$ cuentas/u.tiempo) (Fig.3), ratios de normalización $\left(\mathrm{L}_{\mathrm{N}} / \mathrm{T}_{\mathrm{N}}\right)$ bajos $(<5$ mediante el método de integración LBG y $<10$ mediante el método EBG). El error asociado está por encima del 10\% -incrementándose entre un 10 y un $20 \%$ al emplear el método EBG. La señal OSL natural de la única muestra procedente de materiales graníticos (Vilaboa- MPK8) es más intensa (>40.000c/u.t)
(Fig.3), con ratios más elevados y menor incertidumbre -aspecto que se relaciona con una mayor presencia de radioisótopos $\left({ }^{238} \mathrm{U},{ }^{232} \mathrm{Th} \mathrm{y}{ }^{40} \mathrm{~K}\right)$, en micas, circones y microclina, para este tipo de litologías félsicas (BOYLE, 1982; GASCOYNE, 1992). No obstante, no se observa relación entre señal OSL y dosis equivalente (De), ni en los gráficos de dispersión ni en los análisis de correlación (lineal, logarítmica o exponencial) (ARCE-CHAMORRO, 2017). Existe, por tanto, una respuesta muy variable de los materiales analizados, aspecto que afecta del mismo modo a la magnitud del error absoluto o relativo. De forma generalizada, aquellas alícuotas con una mayor dosis (De) no se corresponden necesariamente con una señal OSL más intensa ni con un error asociado mayor. Tampoco existe relación entre una señal OSL natural $\left(\mathrm{L}_{\mathrm{N}}\right)$ más intensa y una ratio de normalización $\left(\mathrm{L}_{\mathrm{N}} / \mathrm{T}_{\mathrm{N}}\right)$ mayor (ARCE-CHAMORRO, 2017).

\begin{tabular}{|l|c|c|c|c|c|c|c|}
\hline Muestras & $\mathbf{N}$ & $\begin{array}{c}\text { DR-Total } \\
(\mathrm{Gy} / \mathrm{ka})\end{array}$ & $\begin{array}{c}\text { DR-Beta } \\
(\mathrm{Gy} / \mathrm{ka})\end{array}$ & $\begin{array}{c}\text { DR-Gamma } \\
(\mathrm{Gy} / \mathrm{ka})\end{array}$ & $\begin{array}{c}\text { DE } \\
(\mathrm{Gy})\end{array}$ & $\begin{array}{c}\text { OD } \\
(\%)\end{array}$ & $\begin{array}{c}\text { OD } \\
\text { (D-Rec.) }\end{array}$ \\
\hline $\begin{array}{l}\text { MPK1 } \\
\text { (Bribes) }\end{array}$ & $59 / 141$ & $1,50 \pm 0,25$ & $0,82 \pm 0,17$ & $0,60 \pm 0,14$ & $98,57 \pm 6,03$ & $35 \pm 5$ & $<5 \%$ \\
\hline $\begin{array}{l}\text { MPK2 } \\
\text { (Mera) }\end{array}$ & $36 / 202$ & $1,83 \pm 0,14$ & $0,54 \pm 0,14$ & $1,37 \pm 0,92$ & $107,89 \pm 10,54$ & $50 \pm 7$ & $<5 \%$ \\
\hline $\begin{array}{l}\text { MPK3 } \\
\text { (Dorneda) }\end{array}$ & $43 / 168$ & $1,21 \pm 0,20$ & $0,65 \pm 0,16$ & $0,56 \pm 0,12$ & $115,20 \pm 7,01$ & $30 \pm 5$ & $<5 \%$ \\
\hline $\begin{array}{l}\text { MPK4 } \\
\text { (Santa Cruz) }\end{array}$ & $51 / 91$ & $0,90 \pm 0,13$ & $0,46 \pm 0,08$ & $0,34 \pm 0,08$ & $94,72 \pm 6,35$ & $39 \pm 5$ & $<5 \%$ \\
\hline $\begin{array}{l}\text { MPK5 } \\
\text { (Perillo) }\end{array}$ & $51 / 125$ & $1,15 \pm 0,07$ & $0,60 \pm 0,09$ & $0,56 \pm 0,09$ & $88,70 \pm 5,67$ & $32 \pm 5$ & $<5 \%$ \\
\hline $\begin{array}{l}\text { MPK6 } \\
\text { (Cecebre) }\end{array}$ & $65 / 188$ & $0,68 \pm 0,10$ & $0,30 \pm 0,07$ & $0,19 \pm 0,07$ & $31,61 \pm 3,28$ & $78 \pm 7$ & $<5 \%$ \\
\hline $\begin{array}{l}\text { MPK7 } \\
\text { (Bastiagueiro) }\end{array}$ & $26 / 219$ & $1,37 \pm 0,27$ & $0,66 \pm 0,22$ & $0,70 \pm 0,15$ & $135,43 \pm 6,85$ & $13 \pm 5$ & $<5 \%$ \\
\hline $\begin{array}{l}\text { MPK8* } \\
\text { (Vilaboa) }\end{array}$ & $26 / 195$ & $1,64 \pm 0,16$ & $0,84 \pm 0,09$ & $0,79 \pm 0,14$ & $139,58 \pm 9,02$ & $28 \pm 5$ & $<5 \%$ \\
\hline
\end{tabular}

Tabla 1. Tasa de radiación natural (DR-Total, DR-BETA y DR-Gamma), Dosis equivalente estimada mediante el modelo CAM (EBG), porcentaje de sobre-dispersión (OD) respecto a la media ponderada del CAM y porcentaje de OD del Dose-Recovery test. (N): Número de alícuotas aceptadas respecto a las analizadas con el SAR. (*): litología granítica -el resto de muestras proceden de litología esquistosa. 
Los motivos de rechazo de alícuotas (entre el 30 y el $50 \%$ de las analizadas) (Tabla 1) están más relacionados con una señal OSL de fondo por encima de lo recomendable (Señal-fondo $£ 1 / 3$ Señal-natural; MURRAY AND WINTLE, 2000) o con niveles de interpolación dentro del umbral de saturación (WINTLE AND MURRAY, 2006) (estimado por convenio en $200 \mathrm{~Gy}$ ) y, en ocasiones, aberraciones en la forma de la curva de crecimiento. También existe un número considerable de alícuotas para cada una de las muestras que no superan los criterios de aceptación del test de reciclaje (entre el $10 \mathrm{y}$ el 30\%), el cual evalúa las variaciones de sensibilidad que afectan a las alícuotas en los distintos tratamientos radiactivos, térmicos y lumínicos a lo largo de los distintos ciclos del SAR (MURRAY AND WINTLE, 2000).

Por su parte, para la alícuotas aceptadas se observan ratios de normalización bajos a dosis elevadas. En base a los modelos físicos a estos niveles de saturación y dosis, se plantea una mayor contribución de la componente OSL media, en detrimento de la componente OSL rápida (DULLER, 2012; FEATERS AND PAGONIS, 2015), aspecto que hipotéticamente minimiza al emplear el método EBG (BALLARINI ET AL., 2007; CUNNINHAM AND WALLINGA, 2010). Efectivamente, mediante el EBG se incrementan las ratios de normalización hasta un $10 \%$, con curvas de crecimiento más amplias y un descenso de la sobre-dispersión (\%OD) -aunque acompañado de un mayor error asociado y menos alícuotas aceptadas. La curva de caída de esta señal natural OSL, la señal OSL del test-dose y la señal OSL del segundo ciclo de regeneración del SAR (30Gy) para todas las muestras, se caracteriza por un rápido descenso (>90\%) transcurrido $1 \mathrm{~s}$ de estimulo, alcanzando posteriormente valores de fondo. Los resultados del Bleaching-test muestran como a partir de $2 \mathrm{~s}$ de blanqueamiento y después de $0.5 \mathrm{~s}$ de estímulo lumínico, todas las señales OSL alcanzaban niveles de fondo (Fig.3), indicando la presencia de esta componente rápida, más deseable.

Todo apunta a que los materiales presentan una sensibilidad muy baja y una respuesta de luminiscencia muy variable, y que estas variaciones se observan mejor a dosis bajas, como el caso del test-dose. Bajo estas premisas, resulta determinante para algunas muestras la presencia de alícuotas caracterizadas por una señal natural OSL más intensa $\left(\mathrm{L}_{\mathrm{N}}\right)$, una mayor ratio de normalización de la señal natural OSL $\left(\mathrm{L}_{\mathrm{N}} /\right.$ $\mathrm{T}_{\mathrm{N}}$ ), buena interpolación a dosis intermedias dentro de la curva de crecimiento, y con un error asociado más asumible $(<10 \%)$. La De estimada por estas alícuotas, además, se aproximan a las estimaciones promedio de las muestras a partir de la media aritmética, la mediana o, lo que resulta más fiable, la media ponderada por el error del modelo CAM (ARCE-CHAMORRO, 2017). Así, para cada muestra, existen alícuotas con una sensibilidad de luminiscencia adecuada que serán, además, las que tengan una mayor contribución en las estimaciones a partir de ese modelo de edad (GALBRAITH ET AL., 1999). Ante la imposibilidad de analizar la respuesta de los granos más sensibles mediante SG, la evaluación de la variación de dosis frente al tamaño de alícuota ofrece resultados en donde se observa un mejor ajuste a la De estimada y menor error para aquellas alícuotas conformadas por 100 granos (ARCE-CHAMORRO, 2017). 
5.2. Sobredispersión y fuentes de incertidumbre.

Los porcentajes de sobre-dispersión (\%OD) para estas muestras son variables y en ocasiones anómalos (Tabla 1). Empleando la señal OSL-EBG y el modelo CAM (en adelante CAM-EBG), se obtienen porcentajes de OD de $13 \pm 5 \%$ para la muestra MPK 7 de Bastiagueiro y de $30 \pm 5 \%$ para la muestra MPK3 de Dorneda, ambas desarrolladas a partir de esquistos, y para la única muestra procedente de materiales graníticos, MPK8 (Vilaboa), la OD es del $28 \pm 5 \%$, todas ellas dentro del umbral recomendado del 20-30\% (OLLEY ET AL., 1999). El resto de muestras presentan valores por encima del 30\% si bien, las estimaciones de la De para todas ellas son similares y coherentes -incluso a partir del método CAM con la señal OSL-LBG, con dosis entre 90 y 145Gy (Tabla 1). Todo ello, a excepción de la muestra MPK6 (de Cecebre) con valores incoherentes (OD>70\% y De=30Gy).
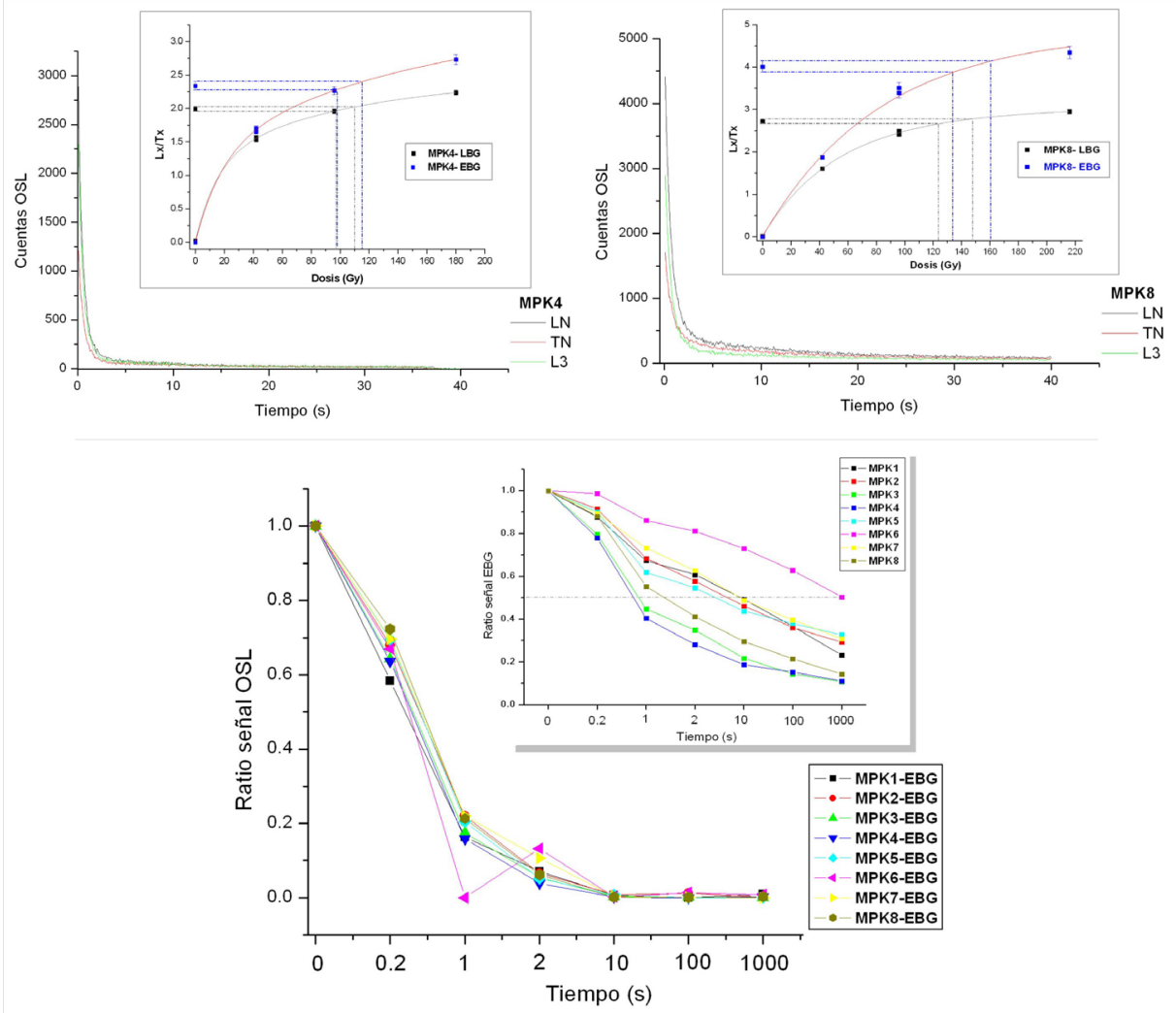

Figura 3. (Arriba): Curva de caída representativa de la señal natural OSL (LN), de la señal OSL del test-dose (TN) y de la señal OSL del 3er ciclo del SAR (L3) de las muestras MPK4 (esquistosa) y MPK8 (granítica). En el recuadro interno se incluye la Curva de Crecimiento de la señal OSL normalizada (LN/TN) mediante el método de integración LBG y EBG para las mismas muestras. (Abajo): Ratio de la Señal OSL normalizada y de la señal de Fondo (EBG) a distintos tiempos de blanqueamiento de la señal OSL mediante el Bleaching-test. 


\subsubsection{Sobredispersión y factores intrínsecos.}

La maximización hipotética de la componente OSL rápida mediante el método EBG se traduce en curvas más amplias, reducción de dispersión por un menor número de alícuotas aceptadas y un mayor error asociado, lo cual no se traduce en diferencias significativas en las estimaciones finales de la De, respecto al método LBG (ARCECHAMORRO, 2017). No obstante, una diferencia muy relevante es la reducción en la estimación de la sobre-dispersión (OD) de hasta un $20 \%$ observado para las muestras (Bastiaguerio-MPK7, Bribes-MPK1, Dorneda-MPK3) (Fig.4) al emplear el método EBG (Tabla 1). Un modo de cuantificar una posible contribución a la sobredispersión de los factores intrínsecos (variaciones en la sensibilidad de luminiscencia de cada alícuota, la respuesta a los distintos tratamientos térmicos y radiactivos en el laboratorio, el comportamiento de la señal OSL-LBG u OSL-EBG en la curva de crecimiento con su error) es a partir de los porcentajes de OD estimados en el test DoseRecovery. A partir de este test, en el que se blanquea completamente la señal y se induce una dosis beta conocida y homogénea, estos factores intrínsecos de incertidumbre son los únicos que podrían afectar en las estimaciones de la De. No obstante, todas las muestras presentan una OD $<5 \%$ (Tabla 1), por lo que se asume que estos factores intrínsecos tienen una contribución a la sobre-dispersión muy escasa, respondiendo a otros factores de naturaleza extrínseca como el blanqueamiento incompleto de la señal o variabilidad en la micro-dosimetría beta del sedimento, y que condicionarán el modelo de edad a utilizar.

\subsubsection{Blanqueamiento de la señal OSL y dis- tribución de datos.}

Todas las distribuciones presentan una dispersión muy amplia. No obstante, en los histogramas no se evidencia la presencia de dos o más poblaciones, presentando un buen ajuste a la normal y una frecuencia acumulada cuasi-sigmoidea (Fig.4). Tampoco se observan datos atípicos relevantes en los diagramas de cajas -a excepción de la muestra anómala MPK6 (ARCE-CHAMORRO, 2017). Los radial-plot evidencian una amplia dispersión, pero en aquellas muestras con menor porcentaje de OD existe un elevado número de alícuotas incluidas dentro del intervalo de confianza en la estimación a partir del modelo CAMEBG (i.c.95\%) (Fig.4). Por su parte, los coeficientes de asimetría y apuntamiento analizados para las estimaciones CAMEBG, incluyendo su error, están dentro de los valores asociados a distribuciones de tipo normal $( \pm 0.5 \%)$ y a partir de los test de bondad de ajuste (K-S (Lillier.) y S.-W) se asume que los datos pertenecen a una distribución de tipo normal ( $\mathrm{p}$-valor $>0.05$ ) (ARCE-CHAMORRO, 2017). Así, el amplio grado de dispersión observada no se asocia a la presencia de dos o más poblaciones por presencia de una señal heredada debido al fenómeno de blanqueamiento incompleto, al tratarse de distribuciones de tipo normal y unimodales. 

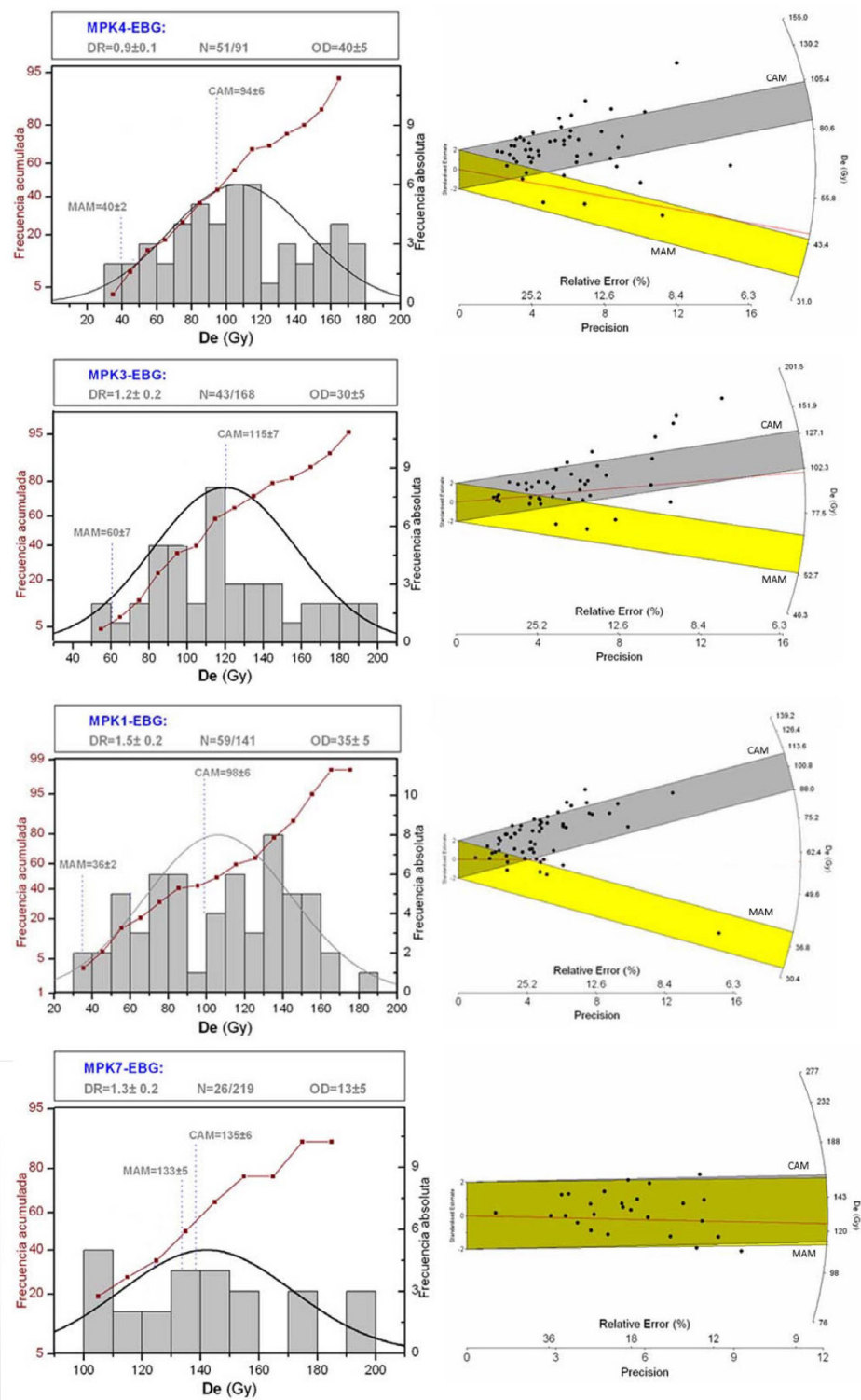

Figura 4. Distribución de las estimaciones: En los histogramas se incluye la frecuencia absoluta, la línea de frecuencia acumulada, el ajuste a una normal, la DR (Gy/ka), el número de alícuotas aceptadas y analizadas con el SAR, la sobre-dispersión (\%OD) y las De (Gy) estimada mediante el CAM y el MAM, para MPK4, MPK3, MPK1 y MPK7. En los Radial-Plot se incluye la precisión y el error relativo (\%) de cada estimación de la De. Los puntos corresponden a cada una de las alícuotas aceptadas. El área gris incluye el i.c. $95 \%( \pm 2 \mathrm{~s})$ del estimador central del CAM-el área amarilla corresponde a este estimador mediante el MAM. 
5.2.3. Sobredispersión y micro-dosimetría beta.

Otro factor extrínseco que puede generar un alto grado de variabilidad y dispersión en las estimaciones de la De está relacionado con la distribución de la radiación beta $\mathrm{D}_{\mathrm{R}}$. BETA en el sedimento muestreado, máxime teniendo en cuenta que el ${ }^{40} \mathrm{~K}$-que emite radiación beta cuando se desintegra en ${ }^{40} \mathrm{Ca}$ - es el radioisótopo que mayor actividad presenta. Así, conociendo la distribución de potasio -con una proporción constante de ${ }^{40} \mathrm{~K}$ (estimada en $11.7 \cdot 10^{-5}$ )- se puede averiguar si este elemento se distribuye de forma homogénea o no en cada una de las fracciones granulométricas y si afecta a la tasa de radiación (DR-TOTAL y DR-BETA) y a la sobre-dispersión (\%OD) (NATHAN ET AL., 2003). Las muestras analizadas se corresponden a áreas concretas incluidas en el sedimento, ricas en materiales detríticos siliciclásticos de tamaño inferior o igual a las arenas $(<2000 \mu \mathrm{m})$, en donde el potasio $(\mathrm{K})$ es minoritario ( $\mathrm{K}<3 \%$; $\mathrm{Fe}>5 \%$; $\mathrm{Al}>20 \%$; $\mathrm{Si}>40 \%)$. Este elemento está aparentemente distribuido de forma homogénea entre las distintas fracciones (finos $(<63 \mu \mathrm{m})$, arenafina $(63-250 \mu \mathrm{m})$, arena-media $(125-500 \mu \mathrm{m})$ y arena-gruesa $(500-1000 \mu \mathrm{m}))$ - a excepción de la muestra granítica de Vilaboa-MPK8, con menor proporción de potasio en la fracción fina, respecto al resto (Fig.5). No obstante, para que la proporción y distribución de potasio en las fracciones ofrezca un dato fiable, es imprescindible calcular la proporción de cada fracción granulométrica. Todas las muestras esquistosas presentan una mayor proporción de limos $(2-63 \mu \mathrm{m})$, con valores entre 30 y $80 \%$, respecto a las arenas. Así, cuando se pondera la proporción de potasio en la fracción fina $(<63 \mu \mathrm{m})$ y gruesa $(63-1000 \mu \mathrm{m})$ con la proporción de estas fracciones en cada muestra, la distribución de este elemento no resulta tan homogénea (Fig.5), con una concentración de $\mathrm{K}$ en la fracción fina muy superior a la gruesa (en proporciones de 5:1 o más), salvo la muestra de Bribes-MPK1. De forma inversa, la muestra origen granítico (Vilaboa-MPK8) presenta una mayor proporción de $\mathrm{K}$ en la fracción gruesa. 


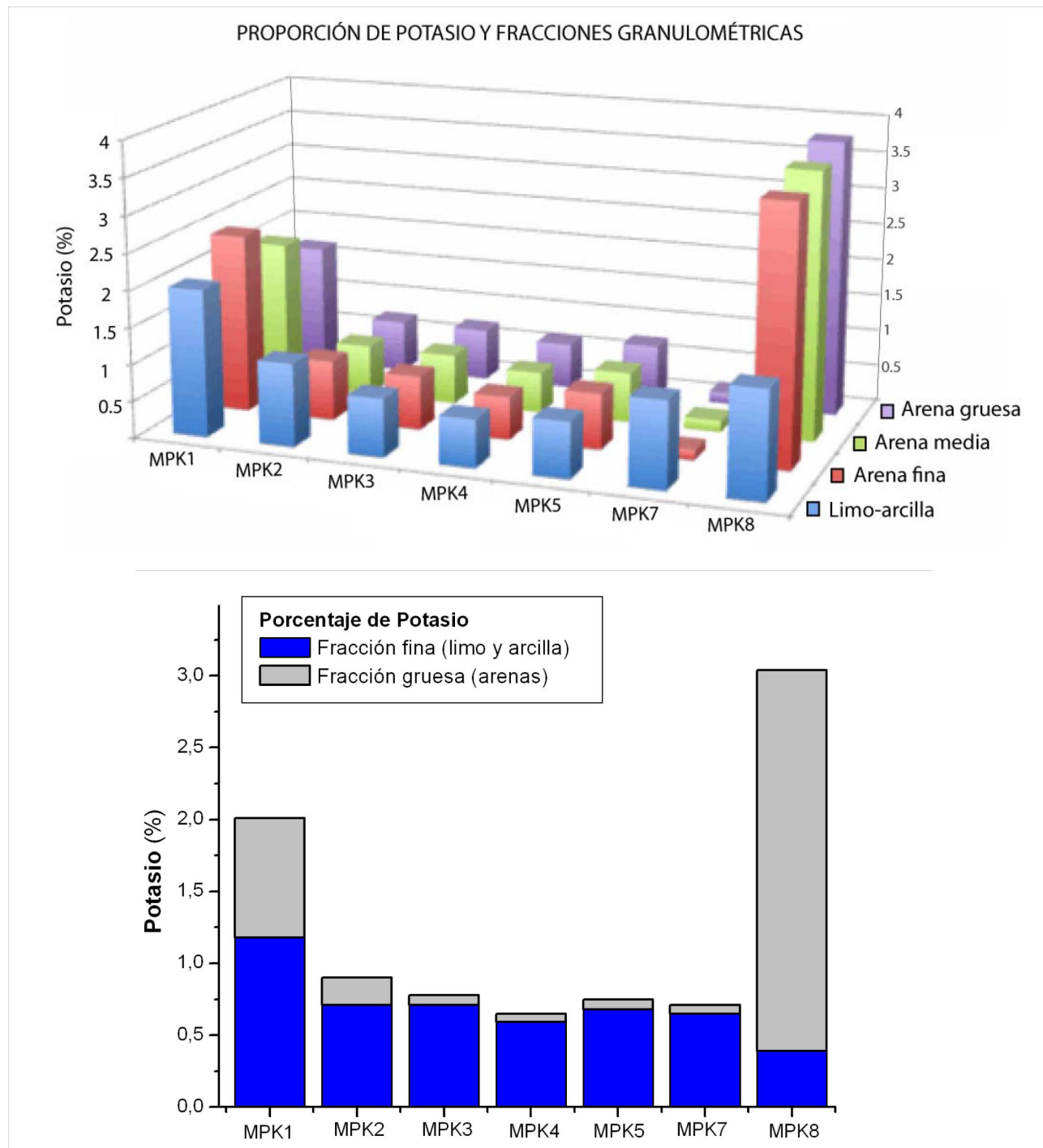

Figura 5. (Arriba): Distribución de Potasio en las fracciones granulométricas (finos $(<63 \mu \mathrm{m})$ y arenas $(63-$ $2000 \mu \mathrm{m})$ ) presentes en la matriz sedimentaria para cada una de las muestras MPK). (Abajo): Porcentaje de potasio ponderado a partir de la proporción de finos y arenas.

En base a los resultados de ajuste lineal entre la proporción de limos y la $\mathrm{D}_{\mathrm{R} \text {-TOTAL }}$ y la $\mathrm{D}_{\text {R-BETA}}$, no se observa una correlación clara, aunque sí una ligera tendencia de sig- no positivo. Esta tendencia continúa cuando se enfrentan los valores de la $\mathrm{D}_{\mathrm{R} \text {-TOTAL }}$ con el porcentaje de $\mathrm{K}$ ponderado en la fracción fina, estableciéndose un nivel de correl- 
ación muy considerable $(\mathrm{R}=0.91)$ cuando se enfrenta con la DR-BETA (Fig.6). Así, un aumento en la proporción de potasio (y por tanto de ${ }^{40} \mathrm{~K}$ ) en la fracción fina, tiene como consecuencia lógica un incremento de la radiación beta. Por otra parte, el ajuste lineal entre la sobre-dispersión (OD) y la DR-TOTAL y la DR-BETA tampoco evidencia una correlación clara $(\mathrm{R}<0.8)$, aunque sí una tendencia significativa (pte. $=0.8$ ) con la componente beta (DR-BETA). Si esta componente está relacionada con la sobre-dispersión y, a su vez, con la proporción de potasio ponderada en finos, el último análisis de correlación lineal no puede ser otro que enfrentar los porcentajes de sobre-dispersión (\%OD) con la proporción de potasio ponderada en la fracción fina. Así, aunque con un coeficiente de correlación bajo $(\mathrm{R}<-0.45)$, sí se observa una tendencia negativa $(\mathrm{pte}=-0.25$ ) a partir de la cual, un incremento en la proporción de potasio en la fracción fina de un $1.0 \%$ a un $2.0 \%$ supone una reducción de los porcentajes de sobre-dispersión desde el 35\% hasta un $8 \%$ (Fig.6). Estos resultados indican que ligeras diferencias en la proporción de potasio están generando una variación en la sobre-dispersión de más del $20 \%$. La variabilidad en la micro-dosimetría beta obser- vada como consecuencia de una distribución heterogénea del potasio podría relacionarse con la naturaleza fluvial de los sedimentos y una elevada probabilidad de lavado y/o lixiviado de los elementos incluidos en estos materiales, retrabajados durante prolongados episodios de transporte y sedimentación (ESCUER-SOLE Y VIDAL-ROMANÍ, 1986; ARCE-CHAMORRO, 2017).

Considerando la escasa contribución de factores intrínsecos al incremento de la misma, la ausencia de blanqueamiento incompleto y la relación observada entre la micro-dosimetría beta con los valores de sobredispersión -así como la propia coherencia del resto de estimaciones (Tabla 1)- cabe aceptar la adecuación del modelo de edad central CAM mediante el método EBG, teniendo como referencia las muestras con menor porcentaje de OD: Bastiaguerio-MPK7 $(\mathrm{OD}=13 \pm 5 \%$; $\mathrm{De}=135 \pm 6 \mathrm{~Gy})$, Vilaboa-MPK8 $(\mathrm{OD}=28 \pm 5 \%$; De $=139 \pm 9 \mathrm{~Gy})$ y DornedaMPK3 (OD $=30 \pm 5 \%$; De=115 \pm Gy) (Tabla 1) -aspecto considerado en otros trabajos de referencia a partir de la señal OSL y aún en presencia de valores de OD 30\% (MEDIALDEA, 2013; TRAUERSTEIN ET AL., 2014; BICKEL ET AL., 2014; HARDT ET AL., 2016; MUÑOZ-SALINAS ET AL., 2017). 


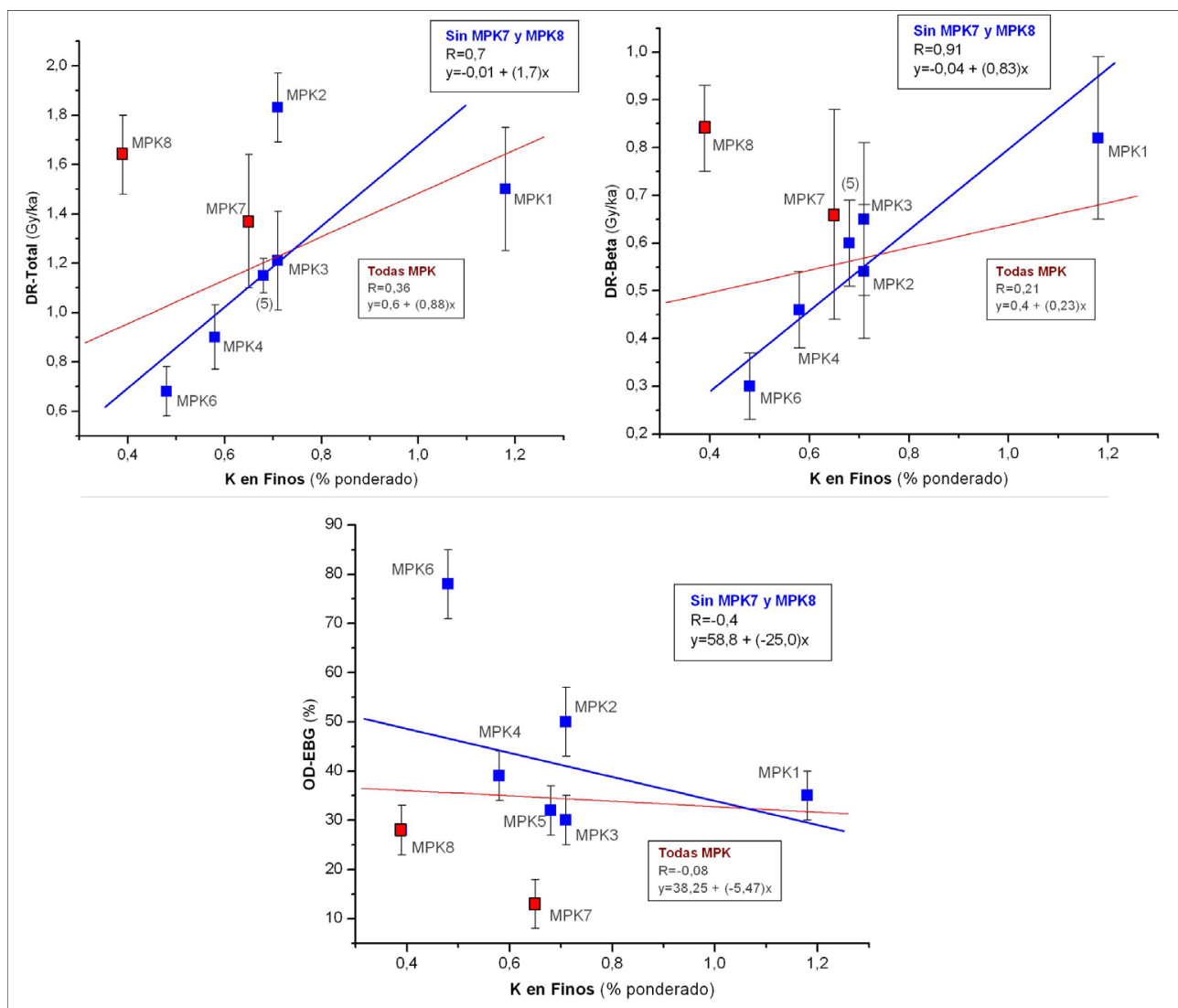

Figura 6. Graficos de correlación lineal: El análisis de correlación se ha llevado a cabo (i) a partir de todas las muestras MPK (línea roja) y (ii) excluyendo la muestra MPK8 (litología granitoide) y MPK7 (sin valores de $\mathrm{OD}$ anómalos) (línea azul). En el eje $\mathrm{X}$ se indica la proporción de Potasio ponderado en finos y en los gráficos se incluye el coeficiente de correlación (R) y la pendiente en la ecuación de la recta para cada caso. (Arriba izq.): Correlación con la DR-Total. (Arriba dcha.): Correlación con la DR-Beta. (Abajo): Correlación con el porcentaje de sobredisperisón (OD) respecto a la media ponderada del CAM-EBG.

\section{CONCLUSIONES}

Los granos de cuarzo incluidos en los sedimentos fluviales analizados para la estimación de la dosis equivalente al tiempo de enterramiento (De) a partir de la señal OSL, están caracterizados por una baja sensibilidad de luminiscencia que se traduce en señales poco intensas, ratios de normalización bajos y un error asociado elevado, siendo, sin embargo, adecuados para la técnica de datación. Estos aspectos justificarían, en parte, la amplia dispersión observada en el conjunto de estimaciones individuales de cada alícuota, pero no justificarían los elevados porcentajes de sobre-dispersión en base a la baja contribución observada por parte de estos factores intrínsecos en el Dose-Recovery test. 
La presencia de distribuciones unimodales, de tipo normal y simétricas, centradas en aquellos valores estimados para la media aritmética, la mediana o la media ponderada por el error del modelo CAM, evidencian la ausencia de blanqueamiento incompleto de la señal OSL que pudieran presentar algunos granos de cuarzo al ser transportados en este medio turbio estudiado, con una elevada carga de limos en suspensión.

Los valores anómalos de sobre-dispersión (OD) están más relacionados con la micro-dosimetría del sedimento, de tal manera que una variación de un $1 \%$ en la proporción ponderada de potasio en finos supone fluctuaciones en el porcentaje de sobre-dispersión del 20\% o más. La distribución desigual del potasio $\left(\mathrm{y}^{40} \mathrm{~K}\right)$, que en el caso de las muestras desarrolladas a partir de litología esquistosa está mucho más concentrado en la fracción fina $(<63 \mu \mathrm{m})$, tiene como consecuencia un reparto heterogéneo de la componente beta $\left(\mathrm{D}_{\text {R-BETA }}\right)$, afectando a la intensidad de la dosis a la que están expuestos los materiales embebidos en la matriz sedimentaria muestreada.

En base a estos análisis, cobra fuerza el modelo de edad central CAM, validándose la estimación de la De para cada muestra.

\section{AGRADECIMIENTOS}

El trabajo con los fluviales del Mero no hubiera sido posible sin la plena colaboración y conocimiento de los mismos del estimado profesor y amigo D.Juan Ramón Vidal Romaní, Catedrático de la UDC y Académico numerario de la RAGCC. Nuestro agradecimiento al Dr.Viveen por su visita al Instituto Universitario de Xeoloxía y sus comentarios en las jornadas de campo reali- zadas en estos afloramientos sedimentarios. También agradecer al Dr. Nuñez-Cardezo los comentarios de los resultados en la Unidad de Análisis Estructural da Universidade da Coruña.

\section{BIBLIOGRAFÍA}

Aitken M.J., Smith, B.W., 1988. Optical dating: recuperation after bleaching. Quaternary Science Reviews 7, 387-394. https://doi.org/10.1016/02773791(88)90034-0

Arce-Chamorro, C., 2017. Datación por luminiscencia de depósitos fluviales y eólicos en el margen occidental de Galicia. Tesis Doctoral. Universidade da Coruña.

Arenas, R., Díaz-García, F., Martínez-Catalán, J.R., Abati, J., González-Cuadra, P., Andonaegui, P., González del Tánago, J., Rubio-Pascual, F., Castiñeiras, P., Gómez-Barreiro, J., 2000. Structure and evolution of the Ordenes Complex. Basement Tectonics 15, Pre-Conference Field Trip. A Coruña, Spain.

Arnold, L.J., Roberts, R.G., 2009. Stochastic modelling of multi-grain equivalent dose (De) distributions: implications for OSL dating of sediment mixtures. Quaternary Geochronology 4, 204-230. https://doi. org/10.1016/j.quageo.2008.12.001

Ballarini, M., Wallinga, J., Wintle, A.G., Bos, A.J.J., 2007. A modified SAR protocol for optical dating of individual grains from young quartz samples. Radiation Measurements 42, 360-369. https:// doi.org/10.1016/j.radmeas.2006.12.016

Bailey, R.M., Smith, B.W., Rhodes, E.J., 1997. Partial bleaching and the decay form characteristics of quartz OSL. Radiation Measurements 27, 123- 
136. https://doi.org/10.1016/S13504487(96)00157-6

Banerjee, D., Botter-Jensen, L., Murray, A.S., 2000. Retrospective dosimetry: estimation of the dose to quartz using the single-aliquot regenerative-dose protocol. Applied Radiation and Isotopes 52, 831-844. https://doi.org/10.1016/S09698043(99)00247-X

Bastida, F., Marcos, A., Marquínez, J., Catalán, J.R., Pérez-Estaún, A., Pulgar, J., 1984. Mapa Geológico de España, E 1:200000, Hoja $n^{\circ} 2-1$, La Coruña.

Bickel, L., Lüthgens, C., Lomax, H., Fiebig, M., 2014. Luminescence dating of glaciofluvial deposits linked to the penultimate glaciation in the Eastern Alps. Quaternary International, 357, 110-124. https:// doi.org/10.1016/j.quaint.2014.10.013

Bøtter-Jensen, L., Thomsen, K.J., Jain, M., 2010. Review of optically stimulated luminescence (OSL) instrumental developments for retrospective dosimetry. Radiation Measurements 45, 253-257. https:// doi.org/10.1016/j.radmeas.2009.11.030

Boyle, R.W., 1982. Geochemical prospecting for thorium and uranium deposits. Elsevier, New York, 489pp.

Brennan, B.J., 2003. Beta doses to spherical grains. Radiation Measurements 37, 299-303. https://doi.org/10.1016/S13504487(03)00011-8

Buckland, C., Bailey, R. , Thomas, D., 2019. Using post-IR IRSL and OSL to date young $(<200$ yrs $)$ dryland aeolian dune deposits. Radiation Measurements, 126;106-131. https://doi.org/10.1016/j. radmeas.2019.106131

Burbidge, C.I., Duller, G.A.T., Roberts, H.M., 2006. De determination for young samples using the standardised OSL response of coarse-grain quartz. Radia- tion Measurements 41, 278-288. https:// doi.org/10.1016/j.radmeas.2005.06.038

CSN, 2000. Proyecto Marna. Mapa de radiación gamma natural. Consejo de Seguridad Nuclear. Madrid.

Cunha, P.P., Martins, A.A., Huot, S., Murray, A., Raposo, L., 2008. Dating the Tejo river lower terraces in the Rodão area (Portugal) to assess the role of tectonics and uplift. Geomorphology 102, 43-54. https://doi.org/10.1016/j.geomorph.2007.05.019

Cunningham, A. C., Wallinga, J., 2010. Selection of integration time intervals for quartz OSL decay curves. Quaternary Geochronology 5, 657-666. https://doi. org/10.1016/j.quageo.2010.08.004

Duller, G. A. T., 2004. Luminescence dating of Quaternary sediments: Recent advances. Journal of Quaternary Science 19, 183-192. https://doi.org/10.1002/ jqs. 809

Duller, G.A.T., 2008. Single-grain optical dating of Quaternary sediments: why aliquot size matters in luminescence dating. Boreas 37, 589-612. https://doi. org/10.1111/j.1502-3885.2008.00051.x

Duller, G.A.T., 2012. Improving the accuracy and precision of equivalent doses determined using the optically stimulated luminescence signal from single grains of quartz. Radiation Measurements 47, 770-777. https://doi.org/10.1016/j.radmeas.2012.01.006

Engels, J.P., 1974. Precambrian complexes in the hercynian of the North-Western Peninsula. Conference Liblice "Precambrian des zones mobiles de 1'Europe". 1972.

Escuer-Sole, J., Vidal-Romaní, J.R., 1987. Facies y modelo local de los depósitos aluviales de la cuenca del río Mero y 
península de Sada (A Coruña, Galicia, NW Spain). Cuaderno do Laboratorio Xeolóxico de Laxe 11, 69-83.

Feathers, J.K., Pagonis, V., 2015. Dating quartz near saturation - Simulations and application at archaeological sites in South Africa and South Carolina. Quaternary Geochronology 30, 416-421. https://doi.org/10.1016/j.quageo.2014.12.008

Galbraith, R.F., Roberts, R.G., Laslett, G.M., Yoshida, H., Olley, J.M., 1999. Optical dating of single and multiple grains of quartz from Jinmium rock shelter, northern Australia: Part I, experimental design and statistical models. Archaeometry 41, 339-364. https://doi. org/10.1111/j.1475-4754.1999.tb00987.x

Gascoyne, M., 1992. Geochemistry of the actinides and their daughters. In: Ivanovich, M., Harmon, R.S. (eds.) Uranium-series disequilibrium: applications to Earth, marine, and environmental sciences. Clarendon Press, Oxford, pp. 34-61.

Hardt, J., Lüthgens, C., Hebenstreit, R., Böse, M., 2016. Geochronological (OSL) and geomorphological investigations at the presumed Frankfurt ice marginal position in northeast Germany. Quaternary Science Reviews 154, 85-99. https://doi. org/10.1016/j.quascirev.2016.10.015

Heer, A., Adamiec, G., Moska, P., 2012. How many grains are there on a single aliquot? Ancient TL. 30. 9-16.

Ivanovich, M., Harmon, R.S. (eds) 1982. Uranium series desequilibrium: Applications to Earth, Marine and Environmental Sciences (2nd Ed.) Clarendon Press, Oxford. 910pp.

Jain, M., Murray, A. S., Bøtter-Jensen, L., 2003. Characterisation of blue-light stimulated luminescence components in different Quartz samples: implications for dose measurement. Radiation Measurements 37, 441-449. https://doi. org/10.1016/S1350-4487(03)00052-0

Martins, A.A., Cunha, P.P., Buylaert J.P., Huot, S., Murray, A.S., Dinis, P., Stokes, M., 2010. K-Feldspar IRSL dating of a Pleistocene river terrace staircase sequence of the Lower Tejo River (Portugal, western Iberia). https://doi. org/10.1016/j.quageo.2009.06.004

Medialdea, A., 2013. Towards the reconstruction of Floyd histories: Luminiescence dating of palaeoflood deposits. Universidad Autónoma de Madrid. Tesis doctoral.

Murray, A.S., Wintle, A.G., 1999. Isothermal decay of optically stimulated luminescence in quartz. Radiation Measurements 30, 119-125. https://doi. org/10.1016/S1350-4487(98)00097-3

Murray, A.S., Wintle, A.G., 2000. Luminescence dating of quartz using an improved single- aliquot regenerative-dose protocol. Radiation Measurements 32, 57-73. https://doi.org/10.1016/S13504487(99)00253-X

Muñoz-Salinas, E., Castillo, M., Caballero. L., Lacan, P., 2017. Understanding landscape dynamics of the Sierra Juarez, southern Mexico: An exploratory approach using inherited luminescence signals. Journal of South American Earth Sciences 76, 208-217. https://doi. org/10.1016/j.jsames.2017.03.001

Nathan, R., Thomas, P.J., Murray, A.S., Rhodes, E.J., 2003. Environmental dose rate heterogeneity of beta radiation and its implications for luminescence dating: Monte Carlo modelling and experimental validation. Radiation Measurements 
37, 305-313. https://doi.org/10.1016/ S1350-4487(03)00008-8

Olley, J. M., Caitcheon, G. G., Roberts, R. G., 1999. The origin of dose distributions in fluvial sediments, and the prospect of dating single grains from fluvial deposits using optically stimulated luminescence. Radiation Measurements 30, 207-217. https://doi.org/10.1016/S13504487(99)00040-2

Oczkowski, H.L., Przegietka, K.R., Lankauf, K.R., Smanda, J.B., 2000. Gamma spectrometry in thermoluminescence dating. Geochronometría, 18. 57-62.

Prescott, J.R., Hutton, J.T., 1994. Cosmic ray contribution to dose rates for luminescence and ESR dating: large depths and long-term time variations. Radiation Measurements 23, 497-500. https:// doi.org/10.1016/1350-4487(94)90086-8

Przegiętka, K., Chruścińska, A., 2013. Analysis of Optical Bleaching of OSL Signal in Sediment Quartz. Radiation Measurements 56, 257-261. https://doi. org/10.1016/j.radmeas.2013.02.009

Ramos, A.M., Cunha, P.P., Cunha, L.S., Gomes, A., Lopes, F.C., Buylaert, J.P., Murray, A.S., 2012. The River Mondego terraces at the Figueira da Foz coastal area (western central Portugal): Geomorphological and sedimentological characterization of a terrace staircase affected by differential uplift and glacio-eustasy. Geomorphology 165-166, 107-123. https://doi.org/10.1016/j.geomorph.2012.03.037

Rhodes, E.J., 2000. Observations of thermal transfer OSL signals in glacigenic quartz. Radiation Measurements 35, 595-602. https://doi.org/10.1016/S13504487(00)00125-6
Ribeiro, H., Pinto De Jesus, A., SanjurjoSánchez, J., Abreu, I., Vidal-Romaní, J.R., Noronha, F., 2019. Multidisciplinary study of the quaternary deposits of the Vila Nova de Gaia, NW Portugal, and its climate significance.J Iber Geol 45,553-563. https://doi.org/10.1007/ s41513-019-00109-9

Rittenour, T.M., 2008. Luminescence dating of fluvial deposits: applications to geomorphic, palaeoseismic and archaeological research. Boreas 37, 613635. https://doi.org/10.1111/j.15023885.2008.00056.x

Roberts, R.G., Spooner, N.A., Questiaux D.G., 1994. Palaeodose underestimates caused by extended duration preheats in the optical dating of quartz. Radiation Measurements 23, 647-653. https://doi. org/10.1016/1350-4487(94)90114-7

Rodnight, H., Duller, G.A.T., Wintle, A.G., Tooth, S., 2006. Assessing the reproducibility and accuracy of optical dating of fluvial deposits. Quaternary Geochronology 1, 109-120. https://doi. org/10.1016/j.quageo.2006.05.017

Sanjurjo-Sánchez, J., Vidal Romaní, J.R., 2011. Luminiescence Dating of Pseudokarst Speleothems: A first approach. Spetroscopy Letters 44, 1-6. https://doi. org/10.1080/00387010.2011.610422

Sanjurjo-Sánchez, J., Vidal Romaní, J.R., 2013. Problemas nuevos y procedimientos de datación por OSL para los sedimentos litorales del NO de la Península Ibérica. VII Jornadas de Geomorfología Litoral, Oviedo, España. Geotemas 14, 47-50.

Singarayer, J.S., Bailey, R.M., 2003. Further investigations of the quartz optically stimulated luminescence components using linear modulation. Radiation 
Measurements 37, 451-458. https://doi. org/10.1016/S1350-4487(03)00062-3

Thomsen, K.J., Murray, A.S., Bøtter-Jensen, L., Jungner, H., 2003. Variation with depth of dose distributions in single grains of quartz extracted from an irradiated concrete block. Radiation Measurements 37, 315-321. https://doi. org/10.1016/S1350-4487(03)00006-4

Thomsen, K.J., Murray, A.S., Bøtter-Jensen, L., 2005. Sources of variability in OSL dose measuremnts using single grains of Quartz. Radiation measurements 39, 47-61. https://doi.org/10.1016/j.radmeas.2004.01.039

Trauerstein, M., Lowick, S.E., Preusser, F., Schlunegger, F., 2014. Small aliquot and single grain IRSL and post-IR IRSL dating of fluvial and alluvial sediments from the Pativilca valley, Peru. Quaternary Geochronology 22, 163-174. https://doi. org/10.1016/j.quageo.2013.12.004

Trinidade, M.J., Prudêncio, M.I., SanjurjoSánchez, J., Vidal-Romaní, J.R., Ferraz, T., Fernández-Mosquera, D., Dias, M.I., 2013. Post-depositional processes of elemental enrichment inside dark nodular masses of an ancient aeolian dune from A Coruña, Northwest Spain. Geologica Acta 11, 231-244. https://doi. org/10.1344/105.000001838
Truelsen, J.L., Wallinga, J., 2003. Zeroing of the OSL signal as function of grain size: investigating bleaching and thermal transfer for a young fluvial sample. Geochronometria 22, 1-8.

Viveen, W., Schoorl, J.M., Veldkamp, A., van Balen, R.T., Vidal-Romaní, J.R., 2013. Fluvial terraces of the northwest Iberian lower Miño River. Journal of Maps, 9:4, 513-522. https://doi.org/10.1 080/17445647.2013.821096

Wallinga, J., Murray, A., Wintle A., 2000. The single-aliquot regenerative-dose (SAR) protocol applied to coarse-grain feldspar. Radiation Measurements 32, 529-533. https://doi.org/10.1016/S13504487(00)00091-3

Wentworth, C.K., 1922. A scale of grade class terms for clastic sediments. Journal of Geology 30, 377-392.

Wintle, A.G., Murray, A.S., 2006. A review of quartz optically stimulated luminescence characteristics and their relevance in single-aliquot regeneration dating protocols. Radiation Measurements 41, 369-391. https://doi.org/10.1016/j.radmeas.2005.11.001

Wintle, A.G., 2008. Luminescence dating: where it has been and where it is going. Boreas 37, 471-482. https://doi. org/10.1111/j.1502-3885.2008.00059.x 
